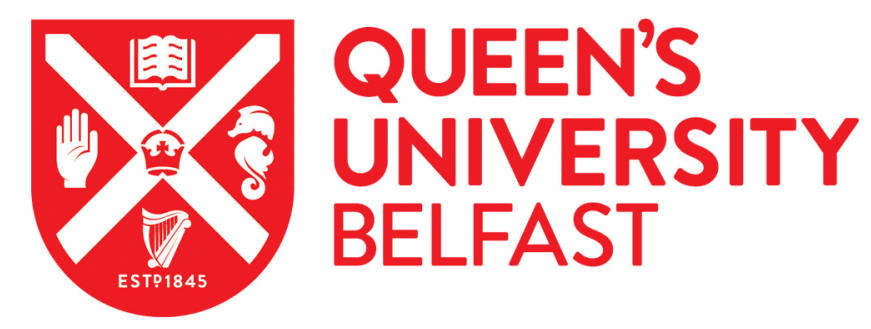

\title{
Aspirin and anti-inflammatory drugs for the prevention of dementia
}

Jordan, F., McGuinness, B., Murphy, K., Passmore, P., Kelly, J. P., \& Devane, D. (2015). Aspirin and antiinflammatory drugs for the prevention of dementia. Cochrane Database of Systematic Reviews, 2015(1), 1-12. https://doi.org/10.1002/14651858.CD011459

Published in:

Cochrane Database of Systematic Reviews

Document Version:

Publisher's PDF, also known as Version of record

Queen's University Belfast - Research Portal:

Link to publication record in Queen's University Belfast Research Portal

Publisher rights

( 2015 The Cochrane Collaboration. Published by John Wiley \& Sons, Ltd. This work is made available online in accordance with the publisher's policies. Please refer to any applicable terms of use of the publisher.

\section{General rights}

Copyright for the publications made accessible via the Queen's University Belfast Research Portal is retained by the author(s) and / or other copyright owners and it is a condition of accessing these publications that users recognise and abide by the legal requirements associated with these rights.

Take down policy

The Research Portal is Queen's institutional repository that provides access to Queen's research output. Every effort has been made to ensure that content in the Research Portal does not infringe any person's rights, or applicable UK laws. If you discover content in the Research Portal that you believe breaches copyright or violates any law, please contact openaccess@qub.ac.uk. 


\section{(E) Cochrane Library}

Cochrane Database of Systematic Reviews

\section{Aspirin and anti-inflammatory drugs for the prevention of dementia (Protocol)}

Jordan F, McGuinness B, Murphy K, Passmore P, Kelly JP, Devane D

Jordan F, McGuinness B, Murphy K, Passmore P, Kelly JP, Devane D.

Aspirin and anti-inflammatory drugs for the prevention of dementia.

Cochrane Database of Systematic Reviews 2015, Issue 1. Art. No.: CD011459.

DOI: 10.1002/14651858.CD011459.

www.cochranelibrary.com 
TABLE OF CONTENTS

HEADER . . . . . . . . . . . . . . . . . . . . . . . . . . . . . . . . . . . . 1

ABSTRACT . . . . . . . . . . . . . . . . . . . . . . . . . . . . . . . . . . . . . . . 1

BACKGROUND . . . . . . . . . . . . . . . . . . . . . . . . . . . . . . . . . . . .

OBJECTIVES . . . . . . . . . . . . . . . . . . . . . . . . . . . . . . . . . . . . . . . . . .

METHODS . . . . . . . . . . . . . . . . . . . . . . . . . . . . . . . . . . . . . . 44

ACKNOWLEDGEMENTS . . . . . . . . . . . . . . . . . . . . . . . . . . . . . . . . . . . . . . . .

REFERENCES . . . . . . . . . . . . . . . . . . . . . . . . . . . . . . . . . . . . . . .

APPENDICES . . . . . . . . . . . . . . . . . . . . . . . . . . . . . . . . . . . . . 9

CONTRIBUTIONS OF AUTHORS . . . . . . . . . . . . . . . . . . . . . . . . . . . . . . . . . . . . . . .

DECLARATIONS OF INTEREST . . . . . . . . . . . . . . . . . . . . . . . . . . . . . . . . 12

SOURCES OF SUPPORT . . . . . . . . . . . . . . . . . . . . . . . . . . . . . . . . . . . . . . . . . 


\title{
[Intervention Protocol]
}

\section{Aspirin and anti-inflammatory drugs for the prevention of dementia}

\author{
Fionnuala Jordan ${ }^{1}$, Bernadette McGuinness ${ }^{2}$, Kathy Murphy ${ }^{1}$, Peter Passmore ${ }^{3}$, John P Kelly ${ }^{4}$, Declan Devane ${ }^{1}$ \\ ${ }^{1}$ School of Nursing and Midwifery, National University of Ireland Galway, Galway, Ireland. ${ }^{2}$ Department of Geriatric Medicine, Belfast \\ Health and Social Care Trust, Belfast, UK. ${ }^{3}$ Centre for Public Health, Queen's University Belfast, Belfast, UK. ${ }^{4}$ Pharmacology and \\ Therapeutics, NUI Galway, Galway, Ireland
}

Contact address: Fionnuala Jordan, School of Nursing and Midwifery, National University of Ireland Galway, Arus Moyola, Newcastle Road, Galway, Ireland. fionnuala.jordan@nuigalway.ie.

Editorial group: Cochrane Dementia and Cognitive Improvement Group.

Publication status and date: New, published in Issue 1, 2015.

Citation: Jordan F, McGuinness B, Murphy K, Passmore P, Kelly JP, Devane D. Aspirin and anti-inflammatory drugs for the prevention of dementia. Cochrane Database of Systematic Reviews 2015, Issue 1. Art. No.: CD011459. DOI: 10.1002/14651858.CD011459.

Copyright (C) 2015 The Cochrane Collaboration. Published by John Wiley \& Sons, Ltd.

\begin{abstract}
A B S T R A C T
This is the protocol for a review and there is no abstract. The objectives are as follows:

The objective of this systematic review is to evaluate the effectiveness of aspirin and other NSAIDs in the primary and secondary prevention of dementia.
\end{abstract}

\section{B A C K G R O U N D}

Dementia is a term used to describe a variety of illnesses which, although diverse in nature, share common clinical manifestations. Typified by pervasive impairment of mental functioning, progressive memory loss, language difficulties, confusion and disorientation, dementia leads to a decline in the skills required to carry out everyday living activities (Coteilli 2012). Symptoms of dementia also affect behaviour and mood (Cohen-Mansfield 2000; Hoe 2005; Hoe 2006; Hoe 2007; Hoe 2009). As the disease progresses, the increasing severity of symptoms has a devastating effect on the quality of life of the person with dementia and their caregivers (World Health Organization 2012). Dementia is a terminal disease that is largely, but not exclusively, a disorder of old age $(O$ Shea 2011). As the disease progresses, the associated increase in frequency and severity of symptoms has a devastating effect on the quality of life of the person with dementia and his or her caregivers (World Health Organization 2012).
While dementia primarily affects older people, there is increasing awareness of cases that start before the age of 65 years. After 65 years, the prevalence of dementia doubles with every five-year increase in age. Current estimates suggest that there are 35.6 million people living with dementia worldwide, and this is forecast to double by 2030 and more than triple by 2050 . Almost twothirds of the dementia population reside in low and middle income countries and this trend is expected to increase. Dementia is an expensive condition, costing an estimated USD 604 billion worldwide in 2010. Low and middle income countries, despite experiencing higher prevalence rates of dementia, account for only $11 \%$ of the total worldwide costs; $89 \%$ of costs are attributed to high income countries. These discrepancies are accounted for by the differences in costs per person across low, middle and high income countries, estimated at USD 868, USD 6827 and USD 32,865 respectively. The cost of informal care constitutes the bulk of costs in the low and middle income countries, while the direct 
costs of social care have a much greater role in the high income countries due to the costs of long term residential and nursing home care in these countries (Wimo 2010). Given the seriousness of the impact of dementia on all associated with the illness and its increasing prevalence and burden of cost, the World Health Organization 2012 has declared dementia a national and world health priority. There is global interest in research into ways of preventing or delaying the onset of dementia.

\section{Description of the condition}

\section{Dementia: main subtypes and pathophysiology}

\section{Alzheimer's disease}

Alzheimer's disease (AD) was first described by Dr Alois Alzheimer, a German psychiatrist and neuropathologist, in 1906. It is now considered to be the most common cause of dementia, accounting for $60 \%$ to $80 \%$ of all cases. A dementia due to $\mathrm{AD}$ affects approximately 15 million individuals worldwide (World Health Organization 2012). It has a prevalence of approximately $1 \%$ among 60 to 64 year olds, increasing to $40 \%$ in people aged 85 years and older (Brookmeyer 1998). AD is a neurodegenerative disorder. It is characterised pathologically by neuritic plaques and neurofibrillary tangles, and clinically by gradually progressive cognitive decline, impairments in activities of daily living, and behavioural and psychological symptoms (Gorelick 2010). As yet, the precise mechanisms underlying the pathogenesis of $\mathrm{AD}$ are not understood fully, but over 25 years ago a hypothesis was first posited that inflammatory processes are an integral part of the degeneration process (Rogers 1988; McGeer 1995; McGeer 1997). Although dementia due to AD typically occurs in later life, there is an extended preclinical stage that is characterised by progressive neuropathological changes. Risk factors for AD are multiple but two of the greatest risk factors are old age and genetics, specifically being a carrier of the APOE e4 allele of the gene for apoplipoprotein E. Other risk factors include history of mild cognitive impairment (MCI), female gender, cardiovascular disease, obesity, diabetes mellitus and low socioeconomic status, quantified as low income and low level of educational attainment (Barnes 2011; Yaffe 2013).

\section{Vascular dementia}

Vascular dementia $(\mathrm{VaD})$ is the second most common type of dementia. It is caused by cerebrovascular disease that directly or indirectly damages the brain structures associated with cognitive functioning (Roh 2014). Two factors are necessary for a diagnosis of
$\mathrm{VaD}$. These are (a) a cognitive disorder evident on neuropsychological testing, and (b) a history of clinical stroke or cerebrovascular disease detected by neuroimaging which is plausibly linked to the cognitive disorder. $\mathrm{VaD}$ can be classified into 1 . multi-infarct dementia, 2. strategic infarct dementia, 3. haemorrhagic dementia, 4. subcortical ischaemic vascular dementia (SIVD), and 5 . other forms of $\mathrm{VaD}$. The first three types of $\mathrm{VaD}$ may present with sudden onset or sudden exacerbations due to acute cerebrovascular events. Their specific symptoms depend on the cortical or subcortical regions affected. The classic clinical picture is of stepwise progression, where periods of stability are interrupted by periods of rapid decline, fluctuation of symptoms and the presence of focal neurological signs (Pendlebury 2009). In contrast, SIVD is associated with an insidious onset and gradual cognitive decline, mimicking the course of AD (Chui 2007. It is caused by stenosis and occlusion of small vessels, which results in white matter ischaemia and multiple lacunar infarcts in subcortical structures. Finally, other forms of $\mathrm{VaD}$ have heterogeneous causes (for example vasculitis, cerebral amyloid angiopathy, and hereditary diseases such as cerebral autosomal dominant arteriopathy with subcortical infarcts and leukoencephalopathy (CADASIL) (Seo 2007; Park 2013; Patel 2013). Risk factors for $\mathrm{VaD}$ are also multifactorial and include advancing age, male gender, history of cardiovascular disease, diabetes, obesity and smoking (McCullagh 2001).

\section{Mixed dementia}

It has become increasingly apparent that mixed pathologies of dementia subtypes exist, particularly in older patients. Post-mortem results of 1050 people with dementia showed that $86 \%$ revealed Alzheimer-related pathology but only $42.8 \%$ exhibited 'pure' AD, with additional cerebrovascular lesions in $22.6 \%$ and Lewy body pathology in 10.8\% (Jellinger 2006).

\section{Description of the intervention}

\section{Aspirin and other non-steroidal anti-inflammatory drugs}

Aspirin and other non-steroidal anti-inflammatory drugs (NSAIDs) are indicated for the alleviation of pain, inflammation and fever. Due to its anti-aggregation effects on platelets, aspirin is also indicated for the treatment and prevention of cardiovascular disease. Depending on the drug type, dose and duration of treatment, NSAIDs are associated with a variety of adverse effects including gastrointestinal ulcers, serious cardiovascular events, hypertension, acute renal failure and worsening of pre-existing heart failure.

Aspirin (a derivative of salicylate) was the original NSAID. It was introduced onto the market in the late $19^{\text {th }}$ century. Further 
NSAIDs such as indomethacin and ibuprofen began to appear in the 1960s (Conaghan 2012). However, it was not until the early 1970s that the mechanism of action of aspirin (and by extension other NSAIDs) was elucidated by John Vane (Vane 1971). His work identified it as an inhibitor of the enzyme cyclo-oxygenase (COX). COX is responsible for the first stage in the production of prostaglandins, local hormones that have a range of physiological functions. Originally it was thought that all prostaglandin synthesis was initiated by a single form of COX, but now at least two isoforms of COX are known to exist, termed COX-1 and COX-2. COX-1 is present in small amounts in most human tissues and acts as a housekeeping enzyme, involved in the regulation of normal physiological processes such as the maintenance of gastric mucosal integrity, kidney function and platelet aggregation. Conversely, COX-2 is undetectable in most tissues under normal physiological circumstances and is selectively up-regulated after exposure to inflammatory mediators or trauma, contributing to subsequent inflammatory responses and pain.

NSAIDs may be classified according to their selectivity for COX1 or COX-2, or both (Vane 1998). Early NSAIDs, including aspirin, are non-selective COX inhibitors. It was hypothesised that the therapeutic effects (that is analgesic, antipyretic, and anti-inflammatory benefits) of all NSAIDs, including aspirin, were due to inhibition of COX-2 and that unwanted side effects were due to inhibition of COX-1 (Vane 1998). Hence it was thought that if selective COX-2 inhibitors could be developed, such compounds should have a similar efficacy to non-selective NSAIDs but with an improved safety profile as they would allow the continued production of prostaglandins in locations such as the gastrointestinal tract and thus limit adverse effects such as mucosal ulceration (Hawkey 1999). Several COX-2 selective inhibitors were subsequently developed and marketed, including meloxicam, nimesulide and the even more highly selective COX-2 inhibitors known collectively as the coxibs. However, some of these highly selective compounds (namely rofecoxib and valdecoxib) have subsequently been withdrawn from the market for safety reasons, due to increased cardiovascular risk. Such compounds are also not devoid of gastrointestinal problems, although the risk is less than with non-selective NSAIDs (Patricio 2013).

Besides their well-defined effects on the COX isoforms, certain NSAIDs have been proposed to have an effect on the formation of amyloid-beta $(\mathrm{A} \beta)$, the major component of the plaques associated with $\mathrm{AD}$. The synthesis of $\mathrm{A} \beta$ requires the enzyme $\gamma$-secretase. Inhibition of this enzyme is an actively pursued disease-modifying target for AD (Ozudogru 2012), particularly if it can be achieved by not interfering with the processing of other substrates for the enzyme thereby conferring a selectivity of action (Crump 2013). Drugs with such properties are known as g-secretase modulators (GSMs). However, the NSAIDs that possess this GSM property (namely ibuprofen, fluribiprofen, indomethacin and sulindac) are weak in vitro inhibitors of the enzyme and have low brain penetrability (Crump 2013) thus putting into question the relevance of this property in a clinical setting.

\section{How the intervention might work}

In recent years, a well-defined neuroinflammatory response has been identified in Alzheimer's disease with considerable inflammation observed around the plaques and tangles that represent the core histological features of the disease. COX enzymes and their influence on prostaglandin pathways have thus come to attention as a possible therapeutic target (McGeer 2000; Cudaback 2014). The anti-inflammatory properties of aspirin and other NSAIDs may interrupt or prevent inflammatory processes which are important in pathogenesis, thus preventing the onset of AD (Etminan 2003). Moreover, the effect on the production of $\mathrm{A} \beta$ represents another possible mechanism of reducing one of the principal pathological hallmarks of $\mathrm{AD}$, but with the caveats mentioned previously. Aspirin and other NSAIDs may prevent $\mathrm{VaD}$ via their anti-inflammatory and anti-platelet effects (Devine 2003).

\section{Why it is important to do this review}

A considerable body of research has accumulated that investigates whether aspirin and other NSAIDs may have a role in the prevention or management of dementia due to $\mathrm{AD}$ and $\mathrm{VaD}$. Evidence to support such use of NSAIDs stems largely from epidemiological studies. Epidemiolgical studies report a lower prevalence of $\mathrm{AD}$ in patients who have regularly taken NSAIDs for the treatment of rheumatological disorders, suggesting that NSAIDs may have a protective effect against AD (McGeer 1996; Stewart 1997; In'd Veld 2001). Reviews of epidemiological studies also suggest that the protective effects may be influenced by the type and duration of NSAID use. Long term use (defined as greater than 24 months) was associated with greater risk reduction than short term use (defined as less than 1 month) or intermediate term use (defined as 1 to 24 months) (Etminan 2003; Szekely 2004).

The effectiveness of aspirin and other NSAIDs as treatments for patients with dementia due to $\mathrm{AD}$ and $\mathrm{VaD}$ has been evaluated in Cochrane systematic reviews (Rands 2000; Jaturapatporn 2012). The promising epidemiological evidence has not been reproduced in prospective randomised clinical trials, whose results have been largely disappointing. As yet, there has been no Cochrane systematic review on the effectiveness of aspirin and other NSAIDs in the prevention of dementia. As dementia, particularly $\mathrm{AD}$ or $\mathrm{VaD}$, is such a major health concern worldwide, any intervention that delays or prevents the onset of dementia would have a major public health impact. We therefore considered it important to assess in a systematic review the strength of the evidence on the safety and efficacy of these drugs for the primary and secondary prevention of dementia. 
O B J E C T I VES

The objective of this systematic review is to evaluate the effectiveness of aspirin and other NSAIDs in the primary and secondary prevention of dementia.

\section{METHODS}

\section{Criteria for considering studies for this review}

\section{Types of studies}

We will include only randomised or quasi-randomised, doubleblind trials in which aspirin and other NSAIDs were administered for the primary or secondary prevention of dementia. All doses and durations of treatment with aspirin and other NSAIDs will be considered.

\section{Types of participants}

Participants will be adults living in the community setting without a prior diagnosis of dementia. Participants may be identified as cognitively healthy or with mild cognitive impairment (MCI). A variety of definitions of MCI will be accepted but participants with MCI will only be considered for inclusion if they meet the following criteria: (a) there is objective evidence of cognitive decline greater than expected for age, and (b) no significant impairment in activities of daily living.

\section{Types of interventions}

We will include the following comparisons:

1. aspirin at any dose with placebo;

2. other NSAIDs at any dose with placebo.

\section{Types of outcome measures}

\section{Primary outcomes}

- Incidence of dementia, diagnosed according to standard diagnostic criteria or those appropriate at the time the study was undertaken

- Adverse events, e.g. cardiovascular, gastrointestinal or renal events

- Mortality

\section{Secondary outcomes}

- Cognitive decline from baseline

- Activities of daily living (ADL)

- Health-related quality of life

\section{Search methods for identification of studies}

\section{Electronic searches}

We will search ALOIS (www.medicine.ox.ac.uk/alois), the Cochrane Dementia and Cognitive Improvement Group Specialized Register. The search terms we will use are: aspirin OR "cyclooxygenase 2 inhibitor" OR aceclofenac OR acemetacin OR celecoxib OR dexibruprofen OR dexketoprofen OR diclofenac sodium OR diflunisal OR diflusinal OR etodolac OR etoricoxib OR fenbufen OR fenoprofen OR flurbiprofen OR ibuprofen OR indometacin OR indomethacin OR ketoprofen OR lumiracoxib OR mefenamic OR meloxicam OR nabumetone OR naproxen OR nimesulide OR “anti-inflammatory" OR piroxicam OR sulindac OR tenoxicam OR tiaprofenic acid OR triamcinolone OR NSAIDS OR NSAID.

ALOIS is maintained by the Trials Search Co-ordinator for the Cochrane Dementia Group and contains studies in the areas of dementia prevention, dementia treatment and cognitive enhancement in healthy people. The studies are identified from the following.

1. Monthly searches of a number of major healthcare databases: MEDLINE, EMBASE, CINAHL, PsycINFO and LILACS.

2. Monthly searches of a number of trial registers: ISRCTN; UMIN (Japan's Trial Register); the World Health Organization (WHO) International Clinical Trials Registry Platform portal (which covers ClinicalTrials.gov; ISRCTN; the Chinese Clinical Trials Register; the German Clinical Trials Register; the Iranian Registry of Clinical Trials; and the Netherlands National Trials Register, plus others).

3. Quarterly searches of the Cochrane Central Register of Controlled Trials (CENTRAL).

4. Six-monthly searches of a number of grey literature sources: ISI Web of Knowledge Conference Proceedings; Index to Theses; Australasian Digital Theses.

To view a list of all sources searched for by ALOIS see About ALOIS on the ALOIS website.

Additional separate searches will be run in many of the above sources to ensure that the most up-to-date results are retrieved. The search strategy that will be used for the retrieval of reports of trials from MEDLINE (via the OvidSP platform) can be seen in Appendix 1. 


\section{Searching other resources}

We will review citations of reference lists of included studies identified through the search strategy, described above, for additional studies and assess their suitability for inclusion in the review. We will not apply any language restrictions.

\section{Data collection and analysis}

The methods that will be undertaken in this review were designed in accordance with recommendations described in the Cochrane Handbook for Systematic Reviews of Interventions (Higgins 2011).

\section{Selection of studies}

Two review authors (FJ and DD) will independently screen all titles and abstracts identified from searches to identify those which might meet the inclusion criteria. We will retrieve in full text any papers identified as potentially relevant by at least one author. The same review authors will independently screen full text articles for inclusion or exclusion. Any disagreement will be resolved by discussion or, if necessary, we will consult a third review author (KM or PP). All papers excluded from the review at the full text stage will be listed as excluded studies, with reasons provided in the 'Characteristics of excluded studies' table.

\section{Data extraction and management}

We will design a data extraction form. Two review authors (FJ and $\mathrm{KM}$ ) will independently extract data from eligible studies using this form. Any discrepancies will be resolved through discussion or, if necessary, we will consult with a third review author (BMcG or PP). All extracted data will be entered into RevMan (RevMan 2012) by one review author (FJ), and checked for accuracy against the data extraction sheets by a second review author (KM) working independently. When information regarding any of the included studies is unclear, we will endeavour to contact the authors of the original reports to provide further details.

\section{Assessment of risk of bias in included studies}

Two review authors (FJ and DD) will independently assess risk of bias for each of the included studies using the criteria outlined in the Cochrane Handbook for Systematic Reviews of Interventions (Higgins 2011). We will resolve any disagreements by discussion or, if necessary, we will involve another review author.

\section{(I) Random sequence generation (checking for possible selection bias)}

We will describe for each included study the method used to generate the allocation sequence in sufficient detail to allow an assessment of whether it should produce comparable groups.
We will assess the risk of bias for sequence generation as:

- low risk (any truly random process, e.g. random number table; computer random number generator);

- high risk (any non-random process, e.g. odd or even date of birth; hospital or clinic record number);

- unclear risk.

\section{(2) Allocation concealment (checking for possible selection} bias)

We will describe for each included study the method used to conceal allocation to interventions prior to assignment and will assess whether intervention allocation could have been foreseen in advance of, or during recruitment, or changed after assignment. We will assess the risk of bias for allocation concealment as:

- low risk (e.g. telephone or central randomisation; consecutively numbered sealed opaque envelopes);

- high risk (e.g. open random allocation; unsealed or nonopaque envelopes; alternation; date of birth);

- unclear risk.

\section{(3.I) Blinding of participants and personnel (checking for possible performance bias)}

We will describe for each included study the methods used, if any, to blind study participants and personnel from knowledge of which intervention a participant received. We will consider that studies are at low risk of bias if they were blinded.

We will assess the risk of bias for blinding of participants and personnel as:

- low, high or unclear risk of bias for participants;

- low, high or unclear risk of bias for personnel.

\section{(3.2) Blinding of outcome assessment (checking for possible} detection bias)

We will describe for each included study the methods used, if any, to blind outcome assessors from knowledge of which intervention a participant received.

We will assess the risk of bias for blinding of outcome assessment as:

- low risk;

- high risk;

- unclear risk.

(4) Incomplete outcome data (checking for possible attrition bias due to the amount, nature and handling of incomplete outcome data)

We will describe for each included study, and for each outcome, the completeness of data including attrition and exclusions from the analysis. We will state whether attrition and exclusions were 
reported, the numbers included in the analysis at each stage (compared with the total number of randomised participants), reasons for attrition or exclusion where reported, and whether missing data were balanced across groups or were related to outcomes. Where sufficient information is reported, or can be supplied by the trial authors, we will re-include missing data in the analyses which we undertake. We will assess the risk of bias for incomplete outcome data as:

- low risk (e.g. no missing outcome data; missing outcome data balanced across groups; $<20 \%$ missing data);

- high risk (e.g. frequency of or reasons for missing data that are imbalanced across groups; $>20 \%$ missing data);

- unclear risk.

\section{(5) Selective reporting (checking for reporting bias)}

We will investigate the possibility of selective outcome reporting bias by cross-checking outcomes of interest reported in the methods section to those reported in the results section of the trial publications.

We will assess the risk of bias for selective reporting as:

- low risk (where it is clear that all of the study's pre-specified outcomes and all expected outcomes of interest to the review have been reported);

- high risk (where not all the study's pre-specified outcomes have been reported; one or more reported primary outcomes were not pre-specified; outcomes of interest are reported incompletely and so cannot be used; study fails to include results of a key outcome that would have been expected to have been reported);

- unclear risk.

\section{(6) Other bias (checking for bias due to problems not covered by (I) to (5) above)}

We will describe for each included study any important concerns we have about other possible sources of bias.

We will assess the risk of other forms of bias as:

- low risk;

- high risk;

- unclear risk.

\section{(7) Overall risk of bias}

We will make explicit judgements about whether studies are at high risk of bias, according to the criteria given in theCochrane Handbook for Systematic Reviews of Interventions (Higgins 2011). With reference to (1) to (6) above, we will assess the likely magnitude and direction of the bias and whether we consider it is likely to impact on the findings. We will explore the impact of the level of bias through undertaking sensitivity analyses (see 'Sensitivity analysis') and we will assess the overall risk of bias for each included study as:

- low risk;
- high risk;

- unclear risk.

\section{Measures of treatment effect}

\section{Dichotomous data}

For dichotomous data such as dementia or no dementia, we will present results as summary risk ratios with $95 \%$ confidence intervals $(95 \% \mathrm{CI})$.

\section{Continuous data}

For continuous data, we will use the mean difference with $95 \%$ CI if outcomes are measured with the same scale in all trials. We will use the standardised mean difference with $95 \%$ CI to combine outcomes from trials that measure the same outcome but use different scales (Higgins 2011).

\section{Time-to-event data}

Survival analysis will be considered for time-to-event data and the intervention effect expressed as a hazard ratio. Methods used to analyse time-to-event outcomes will be guided by those described by Tierney et al (Tierney 2007 and detailed in Chapter 7, section 7.7.6. of the Cochrane Handbook for Systematic Reviews of Interventions (Higgins 2011).

For all outcomes we will assess the quality of the evidence contributing to the estimate of the treatment effect using GRADE ratings. For the primary outcomes and the secondary outcomes of cognition and ADL, we will present these assessments in a summary of findings table.

\section{Unit of analysis issues}

\section{Studies with multiple treatment groups}

If a study involves more than two treatment groups, then any treatment groups not relevant to the review objectives will be excluded. If more than two groups are relevant to a single meta-analysis, then groups will be combined to form single experimental and control groups.

\section{Dealing with missing data}

For included studies, we will note levels of attrition. We will explore the impact of including studies with high levels of missing data (we judge this a priori to be greater than $20 \%$ for a primary outcome) in the overall assessment of treatment effect by using sensitivity analysis.

Aspirin and anti-inflammatory drugs for the prevention of dementia (Protocol)

Copyright $\odot 2015$ The Cochrane Collaboration. Published by John Wiley \& Sons, Ltd. 
For all outcomes, we will carry out analyses, as far as possible, on an intention-to-treat basis. If intention-to-treat data are not available in the publications, 'on-treatment' or the data for those who complete the trial shall be sought and indicated as such.

\section{Assessment of heterogeneity}

Where studies are considered similar enough (based on consideration of populations and interventions) to allow pooling of data using meta-analysis, we will assess the degree of heterogeneity by visual inspection of forest plots and by examining the $\mathrm{Chi}^{2}$ test for heterogeneity. We will assess statistical heterogeneity in each meta-analysis using the $\mathrm{T}^{2}, \mathrm{I}^{2}$ and $\mathrm{Chi}^{2}$ statistics. We will regard statistical heterogeneity as substantial if an $\mathrm{I}^{2}$ statistic is greater than $30 \%$ and either the $\mathrm{T}^{2}$ is greater than zero or there is a low $\mathrm{P}$ value (less than 0.10 ) in the $\mathrm{Chi}^{2}$ test for heterogeneity.

Where we identify substantial clinical, methodological or statistical heterogeneity across included studies, we will consider if it is appropriate to report a pooled effect result from the meta-analysis or use a narrative approach to synthesise data. In this event, we will attempt to explore possible reasons for the heterogeneity by grouping studies that have similar populations and interventions.

\section{Assessment of reporting biases}

If there are 10 or more studies in the meta-analysis, we will investigate reporting biases (such as publication bias) using funnel plots. We will assess funnel plot asymmetry visually. If asymmetry is suggested by a visual assessment, we will perform exploratory analyses to investigate it. We acknowledge that publication bias may not be the only cause of funnel plot asymmetry. If a small study effect is apparent, we will explore other potential reasons, for example diversity in methodological quality, bias and genuine heterogeneity in the intervention effect. If justified, we will investigate other possible causes by undertaking a sensitivity analysis (Sterne 2011).

\section{Data synthesis}

We will conduct separate analyses for studies of cognitively healthy participants and studies of participants with MCI.

We will carry out statistical analysis using the Review Manager software (RevMan 2012). We will use fixed-effect model metaanalysis for combining data where it is reasonable to assume that studies are estimating the same underlying treatment effect, that is where participants, interventions and methods are judged sufficiently similar. If there is clinical heterogeneity sufficient to expect that the underlying treatment effects differ between trials, or if substantial statistical heterogeneity is detected, we will use random-effects model meta-analysis to produce an overall summary if an average treatment effect across trials is considered clinically meaningful. The random-effects model summary will be treated as the average range of possible treatment effects and we will discuss the clinical implications of treatment effects differing between trials. If the average treatment effect is not clinically meaningful, we will not combine trials.

If we use random-effects model analyses, the results will be presented as the average treatment effect with $95 \% \mathrm{CI}$ and with the estimates of $\mathrm{T}^{2}$ and $\mathrm{I}^{2}$.

\section{Subgroup analysis and investigation of heterogeneity}

Where data are available, we will conduct an analysis based on relevant and clinically meaningful subgroups such as class of NSAIDs, that is non-selective COX inhibitors and selective COX-2 inhibitors; dose of NSAIDs, low versus high doses of NSAIDs; and duration of use, short term use (less than one month of cumulative use), intermediate term use (one month or more but less than 24 months of cumulative use); and long term use (greater than two years cumulative use).

We will assess subgroup differences by interaction tests available within RevMan (RevMan 2012). We will report the results of subgroup analyses quoting the $\mathrm{Chi}^{2}$ statistic and $\mathrm{P}$ value, and the interaction test $\mathrm{I}^{2}$ value.

\section{Sensitivity analysis}

We will perform sensitivity analyses based on trial quality by repeating our analysis for only those trials judged of 'high quality'. For the purposes of this review, high quality trials will be defined as trials with low risk of bias due to allocation concealment and low risk of bias due to incomplete outcome data.

These sensitivity analyses will also assist in investigating substantial statistical heterogeneity if present (see Assessment of heterogeneity).

\section{A C K NOW LEDGEMENTS}

We thank the Health Research Board, Ireland; The National University of Ireland, Galway; and the Cochrane Dementia and Cognitive Improvement Group for their general support. 


\section{REFERE N C E S}

\section{Additional references}

\section{Barnes 2011}

Barnes DE, Yaffe K. The projected effect of risk factor reduction on Alzheimer's disease prevalence. Lancet Neurology 2011;10(9):819-28.

Brookmeyer 1998

Brookmeyer R, Gray S, Kawas C. Projections of Alzheimer's disease in the united states and public health impact of delaying disease onset. American Journal of Public Health 1998;88:1337-42.

Chui 2007

Chui HC. Subcortical ischaemic vascular dementia.

Neurologic Clinics 2007;25(3):717-40.

\section{Cohen-Mansfield 2000}

Cohen-Mansfield J. Nonpharmacological management of behavioural problems on persons with dementia: The TREA Model. Alzheimer's Care Quaterly 2000;1(4):722-7.

\section{Conaghan 2012}

Conaghan PG. A turbulent decade for NSAIDs: update on current concepts of classification, epidemiology, comparative efficacy, and toxicity. Rheumatology International 2012;32 (6):1491-2.

\section{Coteilli 2012}

Coteilli M, Manenti R, Zanetti O. Reminiscence therapy in dementia: a review. Maturitas 2012;72(3):203-5.

\section{Crump 2013}

Crump CJ, Johnson DS, Li YM. Development and mechanism of $\gamma$-secretase modulators for Alzheimer's disease. Biochemistry 2013;52(19):3197-216.

\section{Cudaback 2014}

Cudaback E, Jorstad NL, Yang Y, Mortine TJ, Keene CD. Therapeutic implications of the prostaglandin pathway in Alzheimer's disease. Biochemical Pharmacology 2014;88(4): $565-72$.

Devine 2003

Devine ME, Rands G. Does aspirin affect outcomes in vascular dementia? A retrospective case-notes analysis. International Journal of Geriatric Psychiatry 2003;18(5): $425-31$.

Etminan 2003

Etminan M, Gill S, Samii A. Effect of non-steroidal anti-inflammatory drugs on risk of Alzheimer's disease: systematic review and meta-analysis of observational studies. BMJ 2003;327(7407):128.

\section{Gorelick 2010}

Gorelick PB. Role of inflammation in cognitive impairment: results of observational epidemiological studies and clinical trials. Annals of the New York Academy of Sciences 2010; 1207: 155-62.

\section{Hawkey 1999}

Hawkey CJ. COX-2 inhibitors. Lancet 1999;353(9149): $307-14$.
Higgins 2011

Higgins JPT, Green S, editors. Cochrane Handbook for Systematic Reviews of Interventions Version 5.1.0 [updated March 2011]. The Cochrane Collaboration, 2011. Available: www.cochranehandbook.org.

Hoe 2005

Hoe J, Katona C, Roch B, Livingston G. Use of the QOL$\mathrm{AD}$ for measuring quality of life in people with severe dementia: the LASER-AD study. Age and Ageing 2005;34 (2):130-5.

Hoe 2006

Hoe J, Hancock G, Livingston G, Orrell M. Quality of life of people with dementia living in care homes. British Journal of Psychiatry 2006;188(5):460-4.

Hoe 2007

Hoe J, Katona C, Orrell M, Livingston G. Quality of life in dementia: care recipient and caregiver perceptions of quality of life in dementia: the LASER-AD study. International Journal of Geriatric Psychiatry 2007;22(10):1031-6.

Hoe 2009

Hoe J, Hancock G, Woods B, Callis D, Orrell M. Changes in the quality of life of people with dementia living in care homes. Alzheimer Disease and Associated Disorders 2009;23 (3):285-90.

In'd Veld 2001

In't Veld BA, Ruitenberg A, Hofman A, Launer LJ, Van Duijin CM, Stijnen T, et al. Nonsteroidal antiinflammatory drugs and risk of Alzheimer's disease. New England Journal of Medicine 2001;345(21):1515-21.

Jaturapatporn 2012 Jaturapatporn D, Isaac MGEKN, McCleery J, Tabet N. Aspirin, steroidal and non-steroidal anti-inflammatory drugs for the treatment of Alzheimer's disease. Cochrane Database of Systematic Reviews 2012, Issue 2. [DOI: 10.1002/14651858.CD006378.pub2]

Jellinger 2006

Jellinger KA. Cliniciopathological analysis of dementia disorders in the elderly--an update. Journal of Alzheimer's Disease 2006;9(3):61-70.

McCullagh 2001

McCullagh C, Craig D, McIllroy P, Passmore P. Risk factors for dementia. Advances in psychiatric treatment 2001;7: 24-31.

\section{McGeer 1995}

McGreer PL, McGeer EG. The inflammatory response system of brain: implications for therapy of Alzheimer and other neurodegenerative diseases. Brain Research Reviews 1995;21(2):195-218

\section{McGeer 1996}

McGeer PL, Schulzer M, McGeer EG. Arthritis and antiinflammatory agents as possible protective factors for Alzheimer's disease: a review of 17 epidemiological studies. Neurology 1996;47(2):425-32. 


\section{McGeer 1997}

McGeer EG, McGeer PL. Innate inflammatory reaction of the brain in Alzheimer disease. Mc Gill Journal of Medicine 1997;3(2):134-41.

\section{McGeer 2000}

McGeer PL. Cyclo-oxygenase-2 inhibitors: rationale and therapeutic potential for Alzheimer's disease. Drugs Aging 2000;17(1):1-11.

\section{O Shea 2011}

O Shea E, Devane D, Murphy K, Cooney A, Casey D, Jordan F, et al. Effectiveness of a structured education reminiscence-based programme for staff on the quality of life of residents with dementia in long-stay units: a study protocol for a cluster randomised trial. Trials 2011, (12):41.

Ozudogru 2012

Ozudogru SN, Lippa CF. Disease modifying drugs targeting b-amyloid. American Journal of Alzheimers Disease and other Dementias 2012;27:296-300.

\section{Park 2013}

Park JH, Seo SW, Kim C, Kim GH, Noh HJ, Kim ST, et al. Pathogenesis of cerebral microbleeds: in vivo imaging of amyloid and subcortical ischemic small vessel disease in 226 individuals with cognitive impairment. Annals of Neurology 2013;73:584-93.

\section{Patel 2013}

Patel B, Lawrence AJ, Chung AW, Rich P, Mackinnon AD, Morris RG, et al. Cerebral microbleeds and cognition in patients with symptomatic small vessel disease. Stroke 2013; 44(2):356-61.

\section{Patricio 2013}

Patricio JPH, Barbosa JP, Ramos RMM, Antunes NFPA, de Mel, PCS. Relative cardiovascular and gastrointestinal safety of non-selective anti-inflammatory drugs versus cyclooxygenase-2 inhibitors. Clinical Drug Investigation 2013;33 (3): 167-83.

\section{Pendlebury 2009}

Pendlebury ST, Rothwell PB. Prevalence, incidence, and factors associated with pre-stroke and post-stroke dementia: a systematic review and meta-analysis. Lancet Neurology 2009;8:1006-18.

\section{Rands 2000}

Rands G, Orrell M. Aspirin for vascular dementia. Cochrane Database of Systematic Reviews 2000, Issue 4. [DOI: 10.1002/14651858.CD001296]

RevMan 2012

The Nordic Cochrane Centre, The Cochrane Collaboration. Review Manager (RevMan) 5.2. Copenhagen: The Nordic Cochrane Centre, The Cochrane Collaboration, 2012.

\section{Rogers 1988}

Rogers J, Luber-Narod J, Styren SD, Civin WH. Expression of immune system-associated antigens by cells of the human central nervous system: relationship to the pathology of
Alzheimer's disease. Neurobiology of Aging 1988;9(4): 339-49.

\section{Roh 2014}

Roh JH, Lee JH. Recent Updates on Subcortical Ischaemic Vascular Dementia. Journal of Stroke 2014;16(1):18-26.

\section{Seo 2007}

Seo SW, Hwa Lee B, Kim EJ, Chin J, Sun Cho Y, et al. Clinical significance of microbleeds in subcortical vascular dementia. Stroke 2007;38:1949-51.

\section{Sterne 2011}

Sterne JAC, Egger M, Mother D. Chapter 10: Addressing reporting biases. In: Higgins JPT, Green $S$ (editors). Cochrane Handbook for Systematic Reviews of Interventions Version 5.1.0 [updated March 2011]. The Cochrane Collaboration. Available: www.cochranehandbook.org 2011.

\section{Stewart 1997}

Stewart WF, Kawas C, Corrada M, Metter EJ. Risk of Alzheimer's disease and duration of NSAI use. Neurology 1997;48(3):626-32.

\section{Szekely 2004}

Szekely, CA, Thorne JE, Zandi PP, EJ M, Messias E, Breitner JC, Goodman SN. Nonsteroidal anti-inflammatory drugs for the prevention of Alzheimer's disease: a systematic review. Neuroepidemiology 2004;23(4):159-69.

Tierney 2007

Tierney JF, Stewart LA, Ghersi D, Burdette S, Sydes MR. Practical methods incorporating summary time to event data into meta-analysis. Trials 2007;8:16. [DOI: 10.1186/ 1745-6215-8-16]

\section{Vane 1971}

Vane JR. Inhibition of prostaglandin synthesis as a mechanism of action for aspirin-like drugs. Nature New Biology 1971;231(25):232-9.

\section{Vane 1998}

Vane JR, Botting RM. Mechanism of action of nonsteroidal anti-inflammatory drugs. The American Journal of Medicine 1998;104(3A):2S-8S.

Wimo 2010

Wimo A, Prince M. World Alzheimer Report 2010: the global economic impact of dementia. Alzheimer's Disease International, London 2010.

World Health Organization 2012 World Health Organization (WHO). Dementia: A Public Health Priority. World Health Organization, 2012.

Yaffe 2013

Yaffe K, Falvey C, Harris T, Newman A, Satterfield S, Koster A, et al. Effects of socioeconomic disparaties on incidence of dementia among biracial older adults: prospective study. BMJ 2013;347:f7051.

* Indicates the major publication for the study 
A P P E N DICES

\section{Appendix I. MEDLINE search strategy}

1. Aspirin/

2. aspirin.ti,ab.

3. "acetylsalicylic acid".ti,ab.

4. “cyclooxygenase 2 inhibitor”.ti,ab.

5. aceclofenac.ti,ab.

6. acemetacin.ti,ab.

7. celecoxib.ti,ab.

8. dexibruprofen.ti,ab.

9. dexketoprofen.ti,ab.

10. diclofenac sodium.ti,ab.

11. diflunisal.ti,ab.

12. diflusinal.ti,ab.

13. etodolac.ti,ab.

14. etoricoxib.ti,ab.

15. fenbufen.ti,ab.

16. fenoprofen.ti,ab.

17. flurbiprofen.ti,ab.

18. ibuprofen.ti,ab.

19. indometacin.ti,ab.

20. indomethacin.ti,ab.

21. ketoprofen.ti,ab.

22. lumiracoxib.ti,ab.

23. mefenamic.ti,ab.

24. meloxicam.ti,ab.

25. nabumetone.ti,ab.

26. naproxen.ti,ab.

27. nimesulide.ti,ab.

28. anti-inflammatory.ti,ab.

29. piroxicam.ti,ab.

30. sulindac.ti,ab.

31. tenoxicam.ti,ab.

32. tiaprofenic acid.ti,ab.

33. triamcinolone.ti,ab.

34. NSAIDS.ti,ab.

35. NSAIDS.ti,ab.

36. *Anti-Inflammatory Agents, Non-Steroidal/

37. Cyclooxygenase Inhibitors/

38. Triamcinolone/

39. Sulindac/

40. Piroxicam/

41. Sulfonamides/

42. Naproxen/

43. Mefenamic Acid/

44. Diclofenacl

45. Cyclooxygenase 2 Inhibitors/

46. Ketoprofen/

47. Indomethacin/

48. Indomethacin/

Aspirin and anti-inflammatory drugs for the prevention of dementia (Protocol)

Copyright $\odot 2015$ The Cochrane Collaboration. Published by John Wiley \& Sons, Ltd. 
49. Ibuprofen/

50. Flurbiprofen/

51. Fenoprofen/

52. Diclofenac/

53. or $/ 1-52$

54. dement*.ti,ab.

55. exp Dementia/

56. (cognition or cognitive).ti,ab.

57. Cognition/

58. Cognition Disorders/

59. MCI.ti,ab.

60. ACMI.ti,ab.

61. ARCD.ti,ab.

62. SMC.ti,ab.

63. CIND.ti,ab.

64. BSF.ti,ab.

65. AAMI.ti,ab.

66. MD.ti,ab.

67. LCD.ti,ab.

68. QD.ti,ab.

69. AACD.ti,ab.

70. MNCD.ti,ab.

71. MCD.ti,ab.

72. ("N-MCI” or “A-MCI" or "M-MCI").ti,ab.

73. ((cognit* or memory or cerebr* or mental*) adj3 (declin* or impair* or los* or deteriorat* or degenerat* or complain* or disturb* or disorder*)).ti,ab.

74. "preclinical AD”.ti,ab.

75. "pre-clinical AD”.ti,ab.

76. (aMCI or MCIa).ti,ab.

77. (“CDR 0.5 " or "clinical dementia rating scale 0.5 ").ti,ab.

78. (“GDS 3” or "stage 3 GDS”).ti,ab.

79. ("global deterioration scale" and "stage 3").ti,ab.

80. "Benign senescent forgetfulness".ti,ab.

81. "mild neurocognit* disorder*”.ti,ab.

82. (prodrom* adj2 (dement* or AD)).ti,ab.

83. (episodic* adj2 memory).ti,ab.

84. Secondary Prevention/ or Primary Prevention/

85. alzheimer*.ti,ab.

86. "healthy elderly".ti,ab.

87. (cognit* adj3 health*).ti,ab.

88. "older adult*".ti,ab.

89. Aged/ or Middle Aged/

90. pensioner*.ti,ab.

91. "senior citizen*”.ti,ab.

92. “community dwelling”.ti,ab.

93. or/54-93

94.53 and 93

95. randomized controlled trial.pt.

96. controlled clinical trial.pt.

97. (Randomized or randomised).ti,ab.

98. (randomly or quasi-random* or quasi-experiment*).ab.

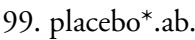

100. groups.ab.

Aspirin and anti-inflammatory drugs for the prevention of dementia (Protocol)

Copyright @ 2015 The Cochrane Collaboration. Published by John Wiley \& Sons, Ltd. 
101. or/95-100

102. (animals not (humans and animals)).sh.

103. 101 not 102

104. 94 and 103

\section{CONTRIBUTIONSOFAUTHORS}

The protocol has been written with FJ as principle author and with contributions from DD, BMcG, PP and KM. Professor John Kelly (JK) advised on the pharmacological aspects of the protocol.

\section{DECLARATIONSOF INTEREST}

None declared

\section{SOURCES OF SUPPORT}

\section{Internal sources}

- National University of Ireland, Galway, Ireland.

\section{External sources}

- Health Research Board, Ireland. 